\title{
Anti-fibrotic effects of Acremoniumterricola milleretal mycelium on immunological hepatic fibrosis in rats
}

\author{
XIA LI $^{1,2^{*}}$, CAN HE $^{1 *}$, WANG-YANG WU ${ }^{1}$, HUAN HUANG $^{1}$, WEI-ZU LI ${ }^{1}$ and YAN-YAN YIN ${ }^{1}$ \\ ${ }^{1}$ Department of Pharmacology, Anhui Medical University, Hefei, Anhui 230032; \\ ${ }^{2}$ Department of Pharmacy, Anhui Province Feidong Country People's Hospital, \\ Feidong, Anhui 231600, P.R. China
}

Received November 6, 2013; Accepted July 23, 2014

DOI: $10.3892 / \mathrm{mmr} .2014 .2604$

\begin{abstract}
Acremoniumterricola milleretal mycelium (AMM) exerts numerous protective effects on organs, and has been used in Chinese herb prescriptions to treat refractory diseases. The aim of this study was to investigate the effects of AMM on immunological hepatic fibrosis induced by porcine serum (PS) in rats. Male Sprague Dawley rats were administered $0.5 \mathrm{ml}$ sterile PS by intraperitoneal injections twice a week for 18 weeks. AMM (175, 350 or $700 \mathrm{mg} / \mathrm{kg})$ and colchicine $(0.1 \mathrm{mg} / \mathrm{kg})$ were administered intragastrically each day until the rats were sacrificed. PS administration resulted in marked hepatic fibrosis, as assessed by increased oxidative stress and hepatic collagen content, as well as $\alpha$-smooth muscle actin $(\alpha$-SMA) expression. AMM significantly reduced liver damage and fibrosis. In addition, AMM decreased the elevation in hydroxyproline, hyaluronic acid, laminin and procollagen type III; increased the activity of superoxide dismutase and glutathione peroxidase; decreased $\alpha$-SMA expression; and eliminated hepatic collagen deposits. Furthermore, AMM inhibited Smad2/3 phosphorylation and Smad7 expression. These results indicate that AMM is able to reduce oxidative stress, inhibit collagen synthesis and block the transforming growth factor- $\beta /$ Smad signaling pathway in a dose-dependent manner.
\end{abstract}

\section{Introduction}

Hepatic fibrosis is a common pathology in China that severely impacts the health of affected individuals and poses a significant risk of morbidity. Fibrosis of the liver

Correspondence to: Professor Yan-Yan Yin, Department of Pharmacology, Anhui Medical University, 81 Meishan Road, Hefei, Anhui 230032, P.R. China

E-mail: yinyanyan5678@126.com

*Contributed equally

Key words: Acremoniumterricola milleretal mycelium, porcine serum, liver fibrosis, transforming growth factor- $\beta / \mathrm{Smad}$ occurs predominantly through the accumulation of collagen, proteoglycans and other macromolecules within the extracellular matrix (ECM). Both the quantity and composition of the ECM are often markedly altered in patients with liver fibrosis $(1,2)$. Chronic liver disease is known to progress from mild to severe inflammation, and then to fibrosis and cirrhosis. Hepatic fibrosis therefore occurs between chronic liver disease and cirrhosis, and constitutes a dynamic and bidirectional stage (3). In the present study, porcine serum (PS)-induced hepatic fibrosis was used as a unique model of liver fibrosis in the absence of obvious hepatocyte injury or inflammatory cell infiltration (4,5). This liver fibrosis model, induced by an immunological reaction, was similar to models generated by repeat injections of equine serum, egg yolk and human albumin, but not rat serum (6). Previous research has indicated that hepatic fibrosis is a complex pathological process involving numerous cytokines and cell signaling pathways (7). The activation of hepatic stellate cells (HSCs), which produce collagen, is considered to be induced in a paracrine manner through the mediation of various factors released from necrotic hepatocytes, Kupffer cells or endothelial cells (8). Domitrovic and Jakovac (9) suggested that liver fibrosis decreased through the inactivation of HSCs under the control of fibrogenic cytokines. Transforming growth factor (TGF)- $\beta 1$ has a pivotal role in liver fibrosis, and can activate HSCs, the principal cellular source of excess ECM during hepatic fibrosis, though the TGF- $\beta /$ Smad signaling pathway (10).

Colchicine has been widely used in clinical practice for the treatment of acute gout and other immunological diseases, and therefore was used as a positive control in the present study. In China, it is believed that patients with liver fibrosis have been treated with herbal medicines for thousands of years. Traditional Chinese medicine is still extensively used for the treatment of liver disease. In recent years, considerable attention has been paid to the use of traditional Chinese medicine in the treatment of liver fibrosis and cirrhosis (11). Acremoniumterricola milleretal mycelium (AMM) is isolated from Acremonium terricola, in submerged fermentation. AMM consists of a number of beneficial components, including Cordyceps polysaccharide, palmitic acid and unsaturated fatty acids, and has been used for its anti-inflammatory and antioxidant effects, as well as for regulation of the immune 
system. This Chinese medicine has been reported to exert various pharmacological effects on animals both in vitro and in vivo (12). Our previous study demonstrated that AMM has protective effects on carbon tetrachloride-induced liver fibrosis in rats (13). The present study was performed to determine whether AMM has any beneficial effects on PS-induced immunological hepatic fibrosis.

\section{Materials and methods}

Materials. AMM was supplied by Anhui Haikui Biotechnology Company (Hefei, China). Colchicine was obtained from Sigma-Aldrich (St. Louis, MO, USA). PS was purchased from Zhengzhou YiKang Biotechnology Company (Zhengzhou, China). Commercial kits used to assay alanine transaminase (ALT), aspartate transaminase (AST), superoxide dismutase (SOD), glutathione peroxidase (GSH-Px) and hydroxyproline (Hyp) were obtained from Nanjing Jiancheng Institute of Biotechnology (Nanjing, China). The hyaluronic acid (HA), laminin (LN) and procollagen type III (PCIII) radioimmunoassay kits were purchased from Beijing North Institute of Biotechnology (Beijing, China). Mouse anti- $\alpha$-smooth muscle actin ( $\alpha$-SMA) and anti- $\beta$-actin antibodies, as well as rabbit anti-phosphorylated- (p-)Smad2, anti-Smad2, anti-p-Smad3, anti-Smad3 and anti-Smad7 antibodies were purchased from Bioworld Technology, Inc., (St. Louis Park, MN, USA).

Animals. Male Sprague Dawley rats, weighing 130-150 g, were provided by the Experimental Animal Center of Anhui Province (Hefei, China). Animals were housed five per cage, with access to water and food ad libitum, and maintained at a constant temperature $\left(22 \pm 1^{\circ} \mathrm{C}\right)$ and humidity $(50 \pm 20 \%)$ under a 12-h light/dark cycle. Animal treatment and maintenance were carried out in accordance with the guidelines for the humane treatment of animals set by the Association of Laboratory Animal Sciences and the Center for Laboratory Animal Sciences, Anhui Medical University (Hefei, China).

Experimental design. After 1 week of acclimation, the animals were subjected to experimentation. Sixty adult male Sprague Dawley rats were randomly divided into six groups, as follows: Control, model (PS-treated), PS plus colchicine $(0.1 \mathrm{mg} / \mathrm{kg})$ and PS plus AMM (175, 350 and $700 \mathrm{mg} / \mathrm{kg}$, respectively). All groups, with the exception of the control, received PS intraperitoneally twice per week for 18 weeks. The colchicine and AMM groups were treated with colchicine $(0.1 \mathrm{mg} / \mathrm{kg}$, intragastrically) and AMM (175, 350 or $700 \mathrm{mg} / \mathrm{kg}$, intragastrically) each day, respectively, at the beginning of the injection of PS. The control and model groups were administered the same volume of vehicle.

The procedure of dividing the animals into groups and for the generation of the PS-induced model of liver fibrosis was based on the method described previously, with certain modifications $(14,15)$. Animals were weighed once per week, and $24 \mathrm{~h}$ after the final injection of PS all animals were sacrificed under anesthesia with ether. Blood samples were collected from the abdominal aorta and centrifuged (3,000 x g for $10 \mathrm{~min})$, and the serum was stored at $-80^{\circ} \mathrm{C}$ until further analysis. The liver was subsequently washed in situ with ice-cold isotonic saline, then removed and divided into two portions; one portion was fixed for histopathology and the other was immediately frozen at $-80^{\circ} \mathrm{C}$ until required.

Hepatotoxicity studies. To assess hepatotoxicity, the serum levels of ALT and AST were measured using commercial kits according to the manufacturer's instructions (Nanjing Jiancheng Institute of Biotechnology).

Hepatic Hyp. The Hyp content in fresh liver samples was measured in accordance with the methods described by Jamall et al (16). The content of hepatic Hyp was determined using a Hyp kit following the manufacturer's instructions (Nanjing Jiacheng Bioengineering Institute).

Oxidative stress. The livers were thawed and washed with normal saline to remove blood and clots. Homogenates were centrifuged $\left(1,000 \mathrm{x} \mathrm{g}\right.$ for $\left.10 \mathrm{~min}, 4^{\circ} \mathrm{C}\right)$ and aliquots of the supernatants were then used to assay the expression levels of SOD and GSH-Px.

Serum fibrotic markers. The serum levels of HA, LN and PCIII were assayed using a radioimmunoassay kit (North Institute of Biotechnology, Beijing, China) according to the manufacturer's instructions.

Histopathology. A portion of the liver specimens of each rat was fixed in $10 \%$ neutral buffered formalin and embedded in paraffin. Sections measuring $4 \mu \mathrm{m}$ were then cut and stained with hematoxylin and eosin (H\&E) and Sirius Red. The stained slides were examined independently by two pathologists with no prior knowledge of their source. The histological grade of hepatic fibrosis was assessed based on the New Inuyama staging system (17): 0, no fibrosis (normal liver and absence of fibrosis); I, fibrosis present (collagen fibers present that extend from the portal triad or the central vein to the peripheral region); II, mild fibrosis (mild collagen fiber presence with extension without compartment formation); III, moderate fibrosis (moderate collagen fibers present with moderate pseudo-lobe formation); IV, severe fibrosis (severe collagen fiber presence with thickening of the partial compartments and frequent pseudo-lobe formation) (18). The percentage of area occupied by collagen fibrosis was calculated by dividing the number of red collagen fibroses by the total number of collagen fibroses. At least five fields were selected under light microscopy at x200 magnification to determine positive collagen fibrosis, and the average was calculated.

Immunohistochemistry. Immunohistochemical analyses were performed on paraffin sections incubated with primary mouse monoclonal $\alpha$-SMA antibody, diluted $1: 100$, at $4^{\circ} \mathrm{C}$ overnight. The sections were then incubated with biotinylated secondary goat anti-mouse immunoglobulin G antibody (Santa Cruz Biotechnology, Inc., Santa Cruz, CA, USA), prior to the sections being counterstained with hematoxylin. Normal mouse antiserum was used as a negative control in place of the primary antibody, and consistently demonstrated no antigenic reaction. The numbers of $\alpha$-SMA-positive and -negative HSCs were visualized using diaminobenzidine (Dako, Tokyo, Japan). Five fields were randomly selected from each section and the number of positive cells for each antibody was counted under 
Table I. Effects of AMM on serum markers of liver damage and antioxidant status in PS-induced hepatic fibrosis.

\begin{tabular}{lccccc}
\hline Group & $\begin{array}{c}\text { Dose (mg/kg) of } \\
\text { AMM/colchicine }\end{array}$ & ALT (U/l) & AST (U/l) & SOD (U/mg) & GSH-Px (U/mg) \\
\hline Control & - & $56.08 \pm 12.26$ & $64.50 \pm 15.76$ & $134.02 \pm 17.23$ & $123.03 \pm 16.18$ \\
PS-treated & - & $67.35 \pm 13.68$ & $81.13 \pm 18.84$ & $97.61 \pm 15.25^{\mathrm{a}}$ & $64.95 \pm 10.89^{\mathrm{a}}$ \\
PS+AMM & 175 & $63.31 \pm 14.43$ & $74.59 \pm 13.52$ & $108.35 \pm 11.05$ & $71.04 \pm 15.78$ \\
& 350 & $61.31 \pm 12.51$ & $71.23 \pm 12.94$ & $117.43 \pm 19.85^{\mathrm{b}}$ & $85.32 \pm 18.06^{\mathrm{b}}$ \\
& 700 & $59.74 \pm 14.62$ & $69.35 \pm 14.12$ & $125.45 \pm 26.21^{\mathrm{b}}$ & $100.73 \pm 17.56^{\mathrm{c}}$ \\
PS+colchicine & 0.1 & $62.16 \pm 15.26$ & $73.36 \pm 17.72$ & $127.67 \pm 22.58^{\mathrm{c}}$ & $83.79 \pm 13.34^{\mathrm{c}}$ \\
\hline
\end{tabular}

${ }^{a} \mathrm{P}<0.001$ vs. the control group; ${ }^{\mathrm{b}} \mathrm{P}<0.05$ and ${ }^{\mathrm{c}} \mathrm{P}<0.01$ vs. the PS- treated group. $\mathrm{n}=8$ per group. Data are presented as the mean \pm standard deviation. AMM, Acremoniumterricola milleretal mycelium; PS, porcine serum; ALT, alanine transaminase; AST, aspartate transaminase; SOD, superoxide dismutase; GSH-Px, glutathione peroxidase.

light microscopy at $\mathrm{x} 400$ magnification. The percentage of $\alpha$-SMA-positive HSCs was calculated by dividing the number of HSCs counted in each slide by the total number of cells, and an average number for each group was calculated.

Western blotting. Frozen liver tissue $(100 \mathrm{mg})$ was washed several times in distilled water and homogenized in extraction buffer (25 mM HEPES, $400 \mathrm{mM} \mathrm{KCl,} 1 \mathrm{mM}$ EDTA and $\left.1.5 \mathrm{mM} \mathrm{MgCl}_{2}\right)$. The homogenate was then centrifuged at $12,000 \times \mathrm{g}$ for $10 \mathrm{~min}$ at $4^{\circ} \mathrm{C}$, and the supernatant was transferred to a fresh tube and stored at $-80^{\circ} \mathrm{C}$. The concentration of protein homogenate was determined using a bicinchoninic acid protein assay kit according to the manufacturer's instructions (ZSGB-BIO, Beijing, China). Proteins were assayed using a Tanon-4200 automatic digital gel imaging system (Tianneng, Shanghai, China) (19). Protein samples (50-150 $\mu \mathrm{g})$ were separated by SDS-PAGE and transferred to polyvinylidene difluoride membranes for $2 \mathrm{~h}$ at $100 \mathrm{~V}$ (Millipore, Bedford, MA, USA). The membranes were then incubated overnight at $4^{\circ} \mathrm{C}$ under agitation with the following primary antibodies (diluted 1:800 in Tris-buffered saline with Tween): Anti-p-Smad2, -p-Smad3, -Smad2, -Smad3, and -Smad7 (Bioworld Technology, Inc.). The membranes were subsequently incubated with secondary antibody (Santa Cruz Biotechnology, Inc.) for $2 \mathrm{~h}$ at room temperature, and the immunoreactive proteins were visualized using enhanced chemiluminescence (Amersham Pharmacia Biotech, Piscataway, NJ, USA) and autoradiography. Gel reverse zymography was performed on the liver of five randomly selected rats per group. Quantity One ${ }^{\circledR}$ software (Bio-Rad, Hercules, CA, USA) was used to quantify the band densities.

Statistical analysis. Data are expressed as the mean \pm standard deviation. Data were analyzed by a one-way analysis of variance, and the Student's t-test was used for two-group comparisons. A Ridit test was used for the statistical analysis of qualitative data. $\mathrm{P}<0.05$ was considered to indicate a statistically significant difference.

\section{Results}

Serum markers of liver damage and antioxidant status. The effects of treatment with AMM on the PS-induced elevation of serum markers due to hepatic damage are shown in Table I. The serum activities of AST and ALT were both increased in the PS-treated group as compared with those in the control group, but the difference did not reach statistical significance (P>0.05). The activities of SOD and GSH-Px in the PS-treated group were significantly lower as compared with those in the control group $(\mathrm{P}<0.001)$. AMM $(350$ and $700 \mathrm{mg} / \mathrm{kg}$ ) only moderately reduced the AST and ALT activities relative to the control group $(\mathrm{P}>0.05)$ but significantly enhanced SOD and GSH-Px activities as compared with the PS-treated group $(\mathrm{P}<0.05$ and $\mathrm{P}<0.01$, respectively) (Table I).

Hepatic Hyp content and serum fibrotic marker levels. The Hyp content and HA, LN and PCIII levels were markedly increased in the PS-treated group as compared with those in the control group $(\mathrm{P}<0.01)$. AMM $(350$ and $700 \mathrm{mg} / \mathrm{kg})$ significantly attenuated the increase in the content of Hyp, and reduced the levels of serum $\mathrm{HA}, \mathrm{LN}$ and PCIII as compared with the PS-treated group $(\mathrm{P}<0.01$ and $\mathrm{P}<0.001)$ (Table II).

Histopathological changes in the liver. The histopathological changes in the liver are shown in Fig. 1. The structure of the liver tissue was normal in the control group, with no detectable changes in the histology (Fig 1A). The livers from the control group stained with Sirius Red stain showed traces of collagen only in the walls of major blood vessels (Fig. 1G). PS administration resulted in an extensive accumulation of collagen in the liver tissue. Fibrosis predominantly developed around the central veins near the hepatic capsule, and complete septal fibrosis was observed; furthermore, pseudo-lobe formation was apparent in severe cases. Only a small number of inflammatory cells were found around the portal area and central vein, without notable hepatocyte necrosis (Fig. 1B and $\mathrm{H}$ ). AMM (350 and $700 \mathrm{mg} / \mathrm{kg}$ ) treatment resulted in a dose-dependent decrease in fibrotic deposits with short fibrous septa, and markedly reduced the pathological changes as compared with the PS-treated group. The structure of the liver tissue remained relatively normal (Fig. 1E, F, K and L).

The PS-treated group showed a high degree of fibrosis. AMM $(350$ and $700 \mathrm{mg} / \mathrm{kg})$ and colchicine $(0.1 \mathrm{mg} / \mathrm{kg})$ treatment resulted in a marked improvement in the histological scores in comparison with the PS-treated group (Table III). 
Table II. Effects of AMM on Hyp content and HA, LN and PCIII levels in PS-induced hepatic fibrosis.

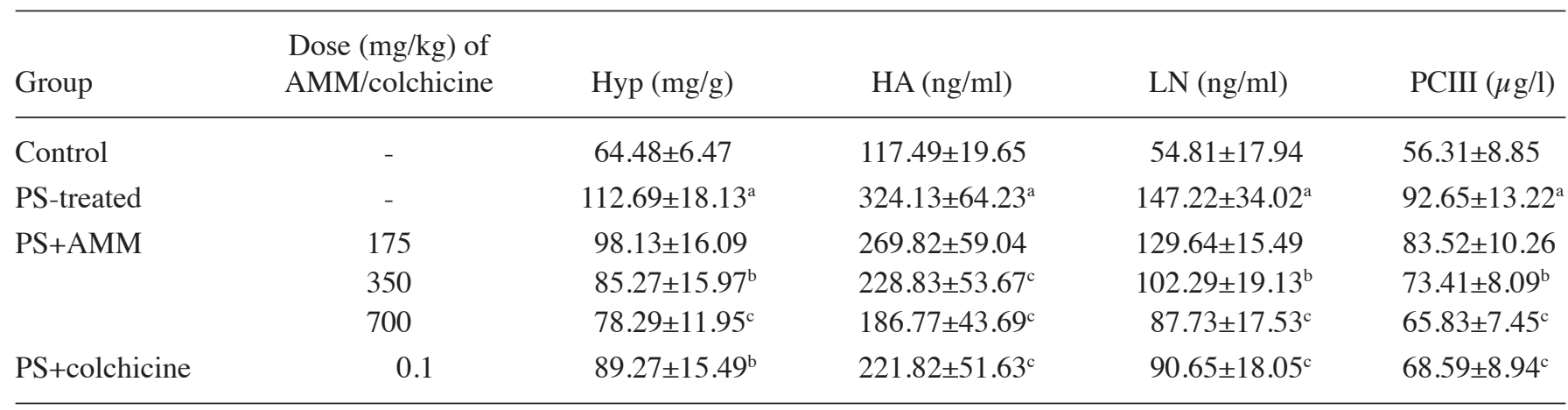

${ }^{\mathrm{a}} \mathrm{P}<0.001$ vs. the control group; ${ }^{\mathrm{b}} \mathrm{P}<0.01$ and ${ }^{\mathrm{c}} \mathrm{P}<0.001$ vs. the PS-treated group. $\mathrm{n}=8$ per group. Data are presented as the mean \pm standard deviation. AMM, Acremoniumterricola milleretal mycelium; PS, porcine serum; HA, hyaluronic acid; Hyp, hydroxyproline; LN, laminin; PCIII, procollagen type III.
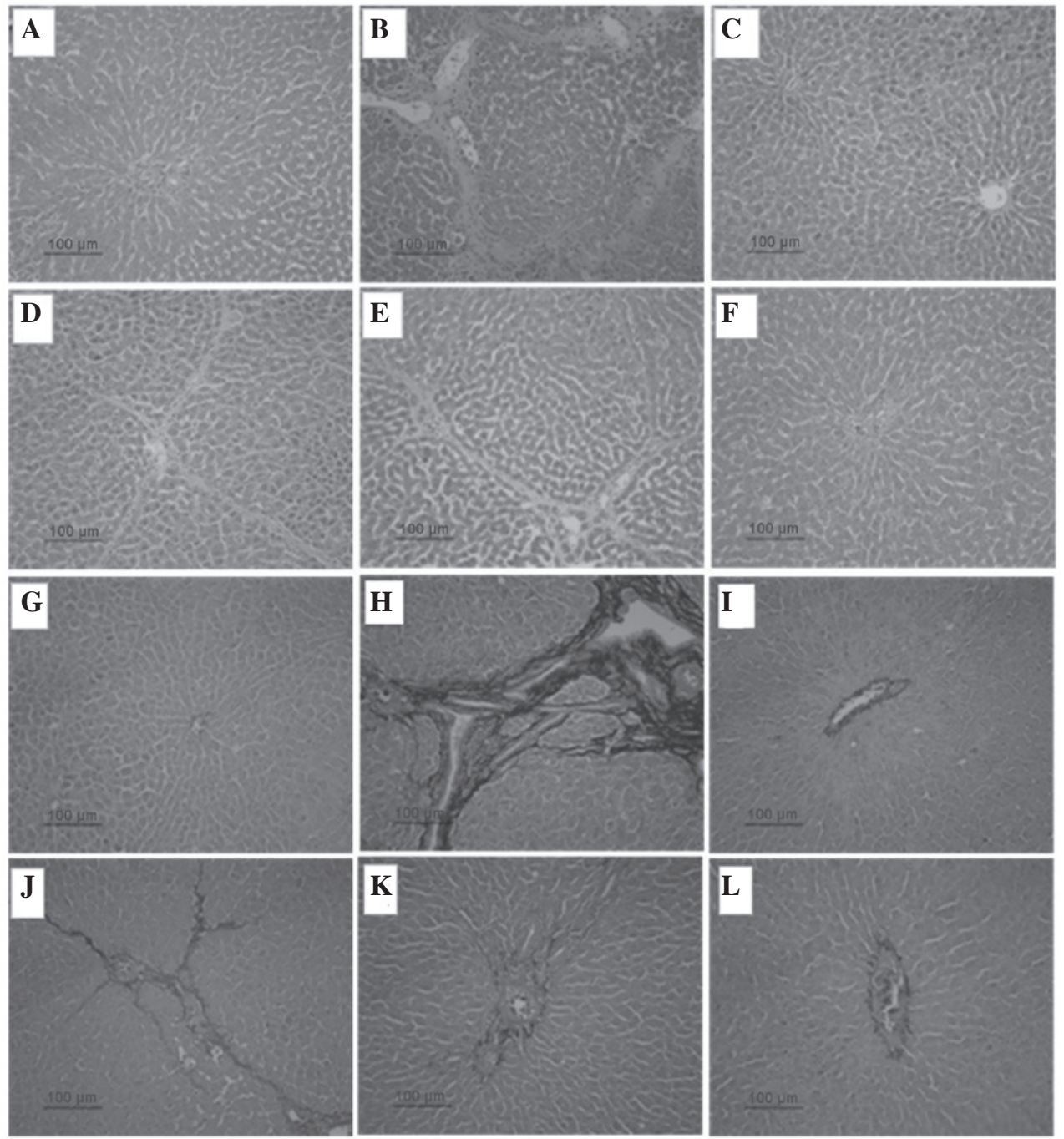

Figure 1. Histological analysis of the effects of AMM on rat liver morphology in PS-induced hepatic fibrosis. Liver tissues were collected and fixed with 10\% formaldehyde. Sections ( $5 \mu \mathrm{m}$ ) were cut and stained with (A-F) hematoxylin and eosin and (G-L) Sirius Red. (A and G) Control group; (B and H) PS-treated group; (C and I) PS and colchicine-treated group (0.1 mg/kg colchicine); (D-F and J-L) PS and AMM-treated groups (175, 350 and $700 \mathrm{mg} / \mathrm{kg} \mathrm{AMM,} \mathrm{respec-}$ tively). Original magnification, x100. AMM, Acremoniumterricola milleretal mycelium; PS, porcine serum.

Immunohistochemical expression of a-SMA. The immunohistochemical expression of $\alpha$-SMA is shown in Fig. 2. Few cells were detected to be positively stained for $\alpha$-SMA in the control group (Fig. 2A). The percentage of $\alpha$-SMA-positive cells was significantly increased in the PS-treated group as compared with that in the control group $(\mathrm{P}<0.001)$, with most 
Table III. Effects of AMM on pathological grading in PS-induced hepatic fibrosis.

\begin{tabular}{lccccccc}
\hline & & \multicolumn{5}{c}{ Pathological grading of hepatic fibrosis (n) } & \\
\cline { 3 - 6 } Groups & $\begin{array}{c}\text { Dose }(\mathrm{mg} / \mathrm{kg}) \text { of } \\
\text { AMM/colchicine }\end{array}$ & 0 & I & II & III & IV & Collagen area (\%) \\
\hline Control & - & 8 & 0 & 0 & 0 & 0 & $1.37 \pm 0.32$ \\
PS-treated & - & 0 & 0 & 1 & 4 & 3 & $10.13 \pm 1.35^{\mathrm{a}}$ \\
PS+AMM & 175 & 0 & 0 & 3 & 3 & 2 & $9.25 \pm 0.83$ \\
& 350 & 0 & 2 & 3 & 3 & 0 & $8.73 \pm 0.79^{\mathrm{b}}$ \\
PS+colchicine & 700 & 0 & 2 & 3 & 2 & 0 & $7.32 \pm 0.57^{\mathrm{c}}$ \\
& 0.1 & 0 & 3 & 3 & 2 & 0 & $8.01 \pm 0.63^{\mathrm{c}}$ \\
\hline
\end{tabular}

Results are the average from 10 fields of vision. ${ }^{a} \mathrm{P}<0.001$ vs. the control group; ${ }^{b} \mathrm{P}<0.05$ and ${ }^{\mathrm{c}} \mathrm{P}<0.001$ vs. the PS-treated group. Data are presented as the mean \pm standard deviation. AMM, Acremoniumterricola milleretal mycelium; PS, porcine serum.
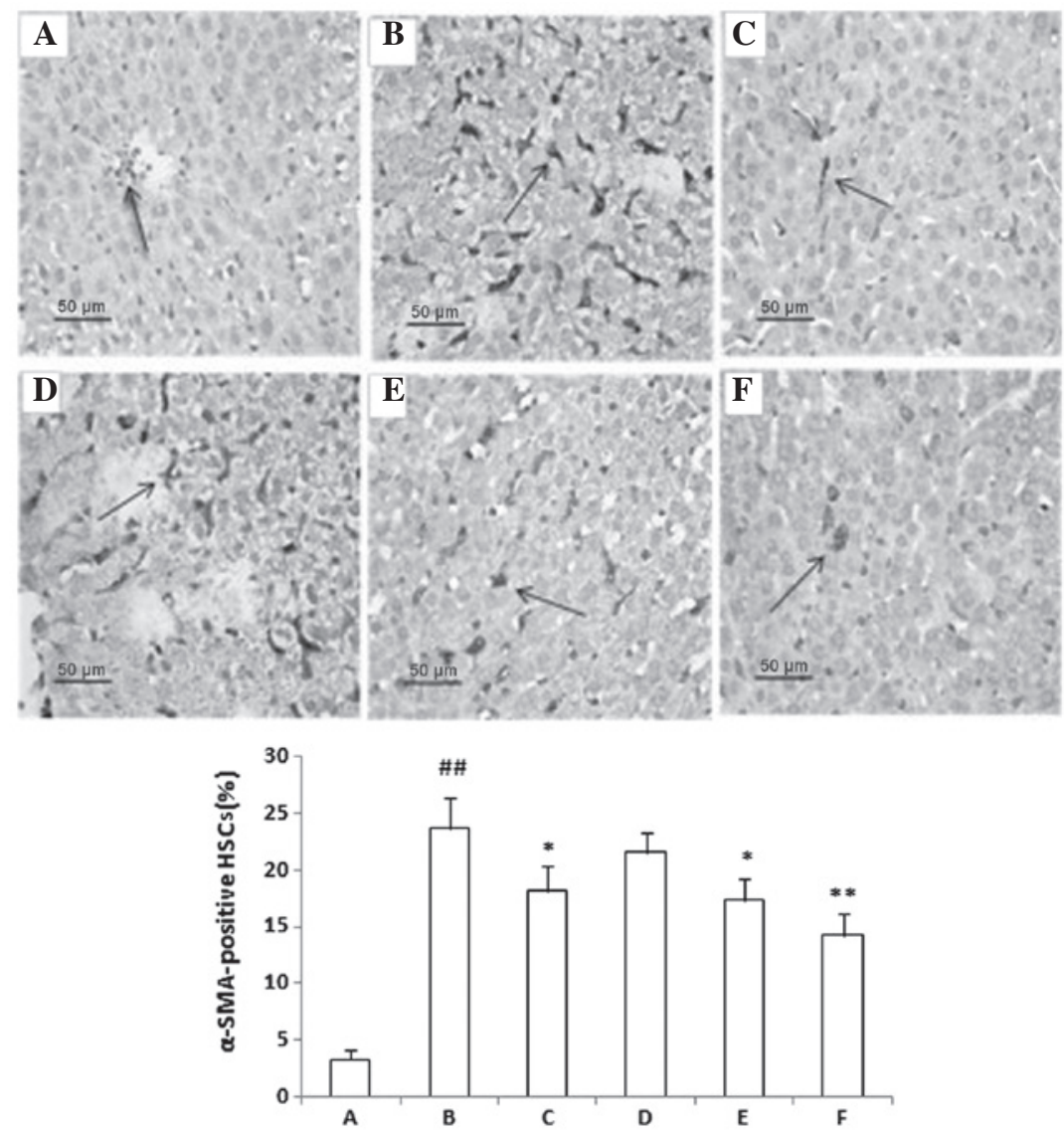

Figure 2. Expression and tissue distribution of $\alpha$-SMA. Representative photomicrographs of five liver sections and a comparison of the number of $\alpha$-SMA-positive HSCs among the groups. (A) Control group; (B) PS-treated group; (C) PS and colchicine-treated group (0.1 mg/kg colchicine); (D-F) PS and AMM-treated groups $\left(175,350\right.$ and $700 \mathrm{mg} / \mathrm{kg}$ AMM, respectively). Arrows show $\alpha-S M A$ immunopositive cells. ${ }^{\# \#} \mathrm{P}<0.001 \mathrm{vs}$. the control group; ${ }^{*} \mathrm{P}<0.01$ and ${ }^{* *} \mathrm{P}<0.001$ vs. the model group. Original magnification, $\mathrm{x} 400$. AMM, Acremoniumterricola milleretal mycelium; PS, porcine serum; $\alpha$-SMA, $\alpha$-smooth muscle actin; HSCs, hepatic stellate cells.

of the $\alpha$-SMA-positive cells detected around the periportal fibrotic band areas, central vein and fibrous septa (Fig. 2B). AMM $(350$ and $700 \mathrm{mg} / \mathrm{kg})$ significantly decreased the percentage of $\alpha$-SMA-positive cells as compared with the PS-treated group $(\mathrm{P}<0.01$ and $\mathrm{P}<0.001$, respectively), with a similar efficacy to that of colchicine $(0.1 \mathrm{mg} / \mathrm{kg})$ (Fig. 2E and F).
Expression of Smad2/3 phosphorylation and Smad7. A higher expression of $\mathrm{p}-\mathrm{Smad} 2 / 3$ was observed in the PS-treated group as compared with the control group $(\mathrm{P}<0.001)$ (Fig. 3A and B). Conversely, the expression of $\mathrm{Smad} 7$ was significantly decreased in the PS-treated group $(\mathrm{P}<0.001)$ (Fig. 3C). AMM (350 and $700 \mathrm{mg} / \mathrm{kg}$ ) and colchicine $(0.1 \mathrm{mg} / \mathrm{kg}$ ) significantly decreased the expression of $\mathrm{p}-\mathrm{Smad} 2$ and $\mathrm{p}-\mathrm{Smad} 3$ 
A
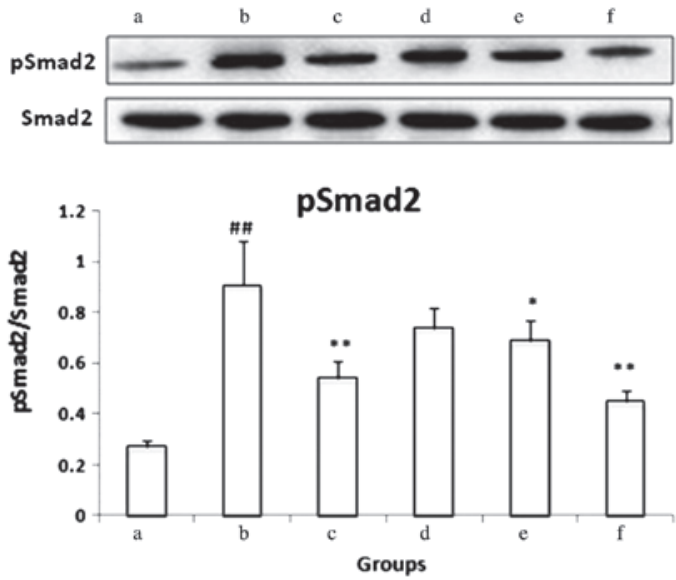

B
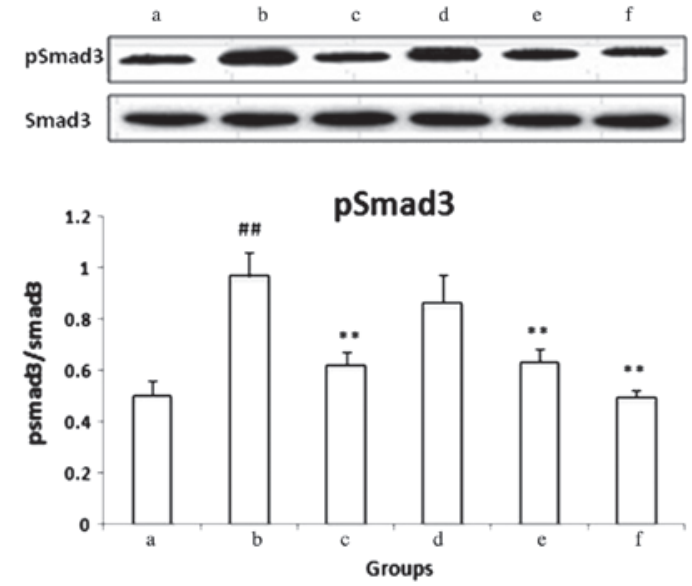

C
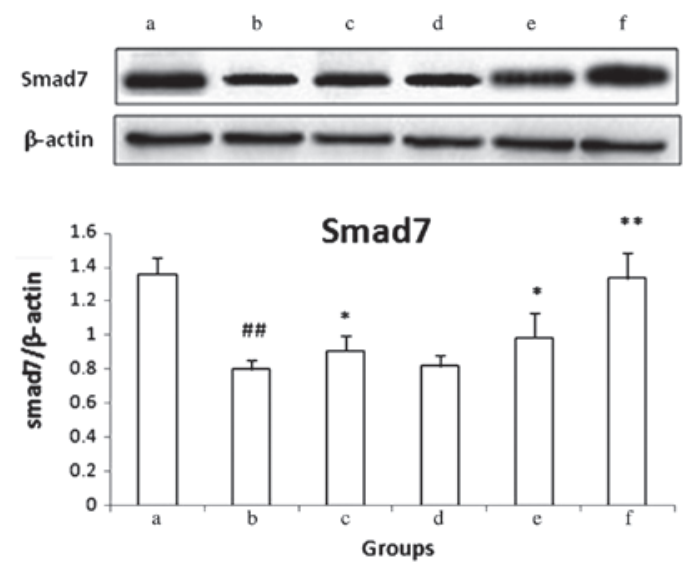

Figure 3. Western blot analysis of (A) p-Smad2, (B) p-Smad3 and (C) Smad7. (a) Control group; (b) PS-treated group; (c) PS and colchicine-treated group $(0.1 \mathrm{mg} / \mathrm{kg}$ colchicine); (d-f) PS and AMM-treated groups $(175,350$ and $700 \mathrm{mg} / \mathrm{kg}$ AMM, respectively). The intensities of p-Smad2, p-Smad3 and Smad7 were normalized to those of total Smad2, Smad 3 and $\beta$-actin, respectively, in the corresponding treatment groups. The presented data are based on at least five independent experiments. Error bars represent the mean \pm standard deviation for five rats in each group. ${ }^{\# /} \mathrm{P}<0.001$ vs. the control group; ${ }^{*} \mathrm{P}<0.05$ and ${ }^{* * *} \mathrm{P}<0.01$ vs. the PS-treated group. AMM, Acremoniumterricola milleretal mycelium; PS, porcine serum; p-Smad, phosphorylated-Smad.

and increased the expression of $\operatorname{Smad} 7(\mathrm{P}<0.05$ and $\mathrm{P}<0.01)$ (Fig. 3A-C). These results demonstrated that AMM (350 and $700 \mathrm{mg} / \mathrm{kg}$ ) may act to prevent hepatic fibrosis by blocking Smad2/3 phosphorylation and enhancing expression of the inhibitor Smad7 in the TGF- $\beta /$ Smad signaling pathway.

\section{Discussion}

Hepatic fibrosis is a common consequence of almost all causes of chronic liver disease. HSCs, the predominant ECM-producing cells, are activated by fibrogenic cytokines, such as TGF- $\beta 1$, angiotensin II and leptin. It was originally believed that hepatic fibrosis was an irreversible process due to disruption of the normal liver architecture (20); however, hepatic fibrosis is more recently considered to be a reversible wound-healing response to chronic liver injury (21). The development of antifibrotic drugs to restrain fibrogenesis is of particular importance. The present study showed by H\&E staining that low numbers of inflammatory cells were present around the portal area and central vein without notable hepatocyte necrosis, in the PS-treated (model) group. This was consistent with the data showing no significant elevation in ALT and AST levels. Sirius Red staining can be used to detect varying degrees of hepatic fibrosis and to score this fibrosis (22). The results showed that $\operatorname{AMM}(350$ and $700 \mathrm{mg} / \mathrm{kg}$ ) markedly attenuated the pathology grading, which was consistent with the findings for Hyp content. SOD and GSH-Px are two primary enzymes of the antioxidant defense system (23). The results presented in this study indicated that AMM could significantly enhance the levels of SOD and GSH-Px. The analysis of Hyp content was used to directly determine the quantity of collagen in the tissue and can be used as a measure to reflect the degree of hepatic fibrosis (24). Additionally, HA, LN and PCIII in serum are considered to be important biomarkers of hepatic fibrogenesis (25). In this study, AMM (350 and $700 \mathrm{mg} / \mathrm{kg}$ ) significantly decreased the Hyp content and the levels of HA, LN and PCIII in PS-induced hepatic fibrotic rats, suggesting that this may be the mechanism underlying its hepatoprotective effects.

HSCs have a key function in the pathogenesis of fibrosis $(26,27)$. Upon the activation of HSCs, levels of fibrillar collagen, particularly types I and II, become markedly increased. $\alpha$-SMA is an indicator of activated HSCs (28). Therefore, the expression level of $\alpha$-SMA was assessed among the groups. Few $\alpha$-SMA-positive HSCs were detected in the AMM (350 and $700 \mathrm{mg} / \mathrm{kg}$ ) treatment groups. Thus, AMM could inhibit the expression of $\alpha$-SMA-positive HSCs and subsequently prevent hepatic fibrosis.

TGF- $\beta 1$ activates HSCs and promotes ECM production, which regulates the fibrogenic process. TGF- $\beta 1$ protein is stored in an inactive form. Once activated, TGF- $\beta 1$ signals via its cognate receptors to Smad proteins (29). The TGF $\beta 1 / \mathrm{Smad}$ signaling pathway has a central role in hepatic fibrosis. Through this pathway, TGF $\beta 1$ predominantly activates HSCs, which can result in hepatic fibrosis $(30,31)$. Activated TGF- $\beta 1$ binds to the constitutively active TGF- $\beta$ Type II (T $\beta$ RII) receptor in the cell membrane, which leads to the recruitment of the T $\beta R I$ receptor. A heterotetrameric complex is formed and results in the phosphorylation and activation of the T $\beta R I$ receptor. Activated T $\beta R I$ in turn phosphorylates Smad2 and Smad3, known as receptor-activated Smads (R-Smads), which form a hetero-oligomeric complex with a common mediator, Smad4 (Co-Smad). This complex translocates to the nucleus, where it can regulate the transcription of target genes (32). Smad7 is an inhibitory Smad (I-Smad) that acts differently to the signal-transducing R-Smads and Co-Smads. Smad7 competitively associates with T $\beta R I$ to prevent the 
phosphorylation of Smad2 and Smad3 and therefore inhibit the signal transduction of TGF $\beta 1$. A previous study showed that Smad7 overexpression inhibited TGF $\beta /$ Smad signaling in rat liver fibroblasts (33). The present study results showed that AMM (350 and $700 \mathrm{mg} / \mathrm{kg}$ ) treatment markedly suppressed the expression of $\mathrm{p}-\mathrm{Smad} 2$, and $\mathrm{p}-\mathrm{Smad} 3$ and increased that of Smad7, indicating that AMM had a significant effect on TGF $\beta /$ Smad signaling.

In conclusion, the present findings indicate that AMM plays an important role in the inhibition of PS-induced immunological hepatic fibrosis. The molecular mechanism of this therapeutic effect could be due to a decrease in oxidative stress, a reduction in the accumulation of collagens and the inhibition of TGF $\beta /$ Smad signaling.

\section{Acknowledgements}

This study was supported by grants from the Nature Science foundation of Anhui Province (nos. KJ2009A031 and KJ2010A164) and The PhD Programs Foundation of Anhui Medical University (no. XJ200821).

\section{References}

1. Dai WJ and Jiang HC: Advances in gene therapy of liver cirrhosis: a review. World J Gastroenterol 7: 1-8, 2001.

2. Mormone E, George J and Nieto N: Molecular pathogenesis of hepatic fibrosis and current therapeutic approaches. Chem Biol Interact 193: 225-231, 2011

3. Pinzani $\mathrm{M}$ and Rombouts K: Liver fibrosis: from the bench to clinical targets. Dig Liver Dis 36: 231-242, 2004.

4. Ochi T, Kawakita T and Nomoto K: Effects of Hochu-ekki-to and Ninjin-youei-to, traditional Japanese medicines, on porcine serum-induced liver fibrosis in rats. Immunopharmacol Immunotoxicol 26: 285-298, 2004.

5. Shiga A, Shirota K, Ikeda T and Nomura Y: Morphological and immunohistochemical studies on porcine serum-induced rat liver fibrosis. J Vet Med Sci 59: 159-167, 1997.

6. Baba Y and Doi K: MHC class II-related genes expression in porcine-serum-induced rat hepatic fibrosis. Exp Mol Pathol 77: 214-221, 2004

7. Seki E, Brenner DA and Karin M: A liver full of JNK: signaling in regulation of cell function and disease pathogenesis, and clinical approaches. Gastroenterology 143: 307-320, 2012.

8. Canbay A, Friedman S and Gores GJ: Apoptosis: the nexus of liver injury and fibrosis. Hepatology 39: 273-278, 2004.

9. Domitrović R and Jakovac H: Effects of standardized bilberry fruit extract (Mirtoselect ${ }^{\circledR}$ ) on resolution of CCl4-induced liver fibrosis in mice. Food Chem Toxicol 49: 848-854, 2011.

10. Friedman SL: Stellate cells: a moving target in hepatic fibrogenesis. Hepatology 40: 1041-1043, 2004.

11. Wang BE: Treatment of chronic liver diseases with traditional Chinese medicine. J Gastroenterol Hepatol 15 Suppl: E67-E70, 2000.

12. Liu Z, Li P, Zhao D, Tang H and Guo J: Protective effect of extract of Cordyceps sinensis in middle cerebral artery occlusion-induced focal cerebral ischemia in rats. Behav Brain Funct 6: 61, 2010

13. Li J, Tian XP, Zhu TJ, Yang LL and Li WJ: Streptomyces fildesensis sp. nov., a novel streptomycete isolated from Antarctic soil. Antonie Van Leeuwenhoek 100: 537-543, 2011.

14. Wu CS, Piao XX, Piao DM, Jin YR and Li CH: Treatment of pig serum-induced rat liver fibrosis with Boschniakia rossica, oxymatrine and interferon-alpha. World J Gastroenterol 11: 122-126, 2005.
15. Andrade RG, Gotardo BM, Assis BC, Mengel $\mathrm{J}$ and Andrade ZA: Immunological tolerance to pig-serum partially inhibits the formation of septal fibrosis of the liver in Capillaria hepatica-infected rats. Mem Inst Oswaldo Cruz 99: 703-707, 2004.

16. Jamall IS, Finelli VN and Que Hee SS: A simple method to determine nanogram levels of 4-hydroxyproline in biological tissues. Anal Biochem 112: 70-75, 1981.

17. Tsai JH, Liu JY, Wu TT, Ho PC, Huang CY, Shyu JC, Hsieh YS, Tsai CC and Liu YC: Effects of silymarin on the resolution of liver fibrosis induced by carbon tetrachloride in rats. J Viral Hepat 15: 508-514, 2008.

18. Kawai K, Uetsuka K, Doi K and Nakayama H: The activity of matrix metalloproteinases (MMPS) and tissue inhibitors of metalloproteinases (TIMPs) in mammary tumors of dogs and rats. J Vet Med Sci 68: 105-111, 2006.

19. Yamada G: Histopathological characteristics and clinical significance of New Inuyama Classification in chronic hepatitis B. Nihon Rinsho 62 Suppl 8: 290-292, 2004 (In Japanese).

20. Popper $\mathrm{H}$ and Uenfriend S: Hepatic fibrosis. Correlation of biochemical and morphologic investigations. Am J Med 49: 707-721, 1970.

21. Toda K, Kumagai N, Kaneko F, Tsunematsu S, Tsuchimoto K, Saito $\mathrm{H}$ and Hibi T: Pentoxifylline prevents pig serum-induced rat liver fibrosis by inhibiting interleukin-6 production. J Gastroenterol Hepatol 24: 860-865, 2009.

22. Hsu YC, Chiu YT, Lee CY, Lin YL and Huang YT: Increases in fibrosis-related gene transcripts in livers of dimethylnitrosamine-intoxicated rats. J Biomed Sci 11: 408-417, 2004.

23. Polavarapu R, Spitz DR, Sim JE, Follansbee MH, Oberley LW, Rahemtulla A and Nanji AA: Increased lipid peroxidation and impaired antioxidant enzyme function is associated with pathological liver injury in experimental alcoholic liver disease in rats fed diets high in corn oil and fish oil. Hepatology 27: 1317-1323, 1998.

24. Dang SS, Wang BF, Cheng YA, Song P, Liu ZG and Li ZF: Inhibitory effects of saikosaponin-d on CCl4-induced hepatic fibrogenesis in rats. World J Gastroenterol 13: 557-563, 2007.

25. Kaneda H, Hashimoto E, Yatsuji S, Tokushige K and Shiratori K: Hyaluronic acid levels can predict severe fibrosis and platelet counts can predict cirrhosis in patients with nonalcoholic fatty liver disease. J Gastroenterol Hepatol 21: 1459-1465, 2006.

26. Choi JH, Hwang YP, Choi CY, Chung YC and Jeong HG: Anti-fibrotic effects of the anthocyanins isolated from the purple-fleshed sweet potato on hepatic fibrosis induced by dimethylnitrosamine administration in rats. Food Chem Toxicol 48: 3137-3143, 2010.

27. Otogawa K, Ogawa T, Shiga R, Ikeda K and Kawada N: Induction of tropomyosin during hepatic stellate cell activation and the progression of liver fibrosis. Hepatol Int 3: 378-383, 2009.

28. Carpino G, Morini S, Ginanni Corradini S et al: Alpha-SMA expression in hepatic stellate cells and quantitative analysis of hepatic fibrosis in cirrhosis and in recurrent chronic hepatitis after liver transplantation. Dig Liver Dis 37: 349-356, 2005.

29. Gressner AM and Weiskirchen R: Modern pathogenetic concepts of liver fibrosis suggest stellate cells and TGF-beta as major players and therapeutic targets. J Cell Mol Med 10: 76-99, 2006.

30. Tsukada S, Westwick JK, Ikejima K, Sato N and Rippe RA: SMAD and p38 MAPK signaling pathways independently regulate alpha1(I) collagen gene expression in unstimulated and transforming growth factor-beta-stimulated hepatic stellate cells. J Biol Chem 280: 10055-10064, 2005.

31. Inagaki Y and Okazaki I: Emerging insights into Transforming growth factor beta Smad signal in hepatic fibrogenesis. Gut 56: 284-292, 2007.

32. Massagué J, Seoane J and Wotton D: Smad transcription factors. Genes Dev 19: 2783-2810, 2005.

33. Kopp J, Preis E, Said H, Hafemann B, Wickert L, Gressner AM, Pallua N and Dooley S: Abrogation of transforming growth factor-beta signaling by SMAD7 inhibits collagen gel contraction of human dermal fibroblasts. J Biol Chem 280: 21570-21576, 2005. 